\title{
Esquistossomose na área hiperendêmica de Taquarendi. I - Infecção pelo Schistosoma mansoni e formas graves
}

\author{
Schistosomiasis in hyperendemic area of Taquarendi. I- Sdistosama mansoni \\ infection and severe clinical forms
}

\author{
José Carlos Bina1 e Aluízio Prata ${ }^{2}$
}

\begin{abstract}
Resumo A localidade de Taquarendi (Bahia) está situada em zona de caatinga, porém com pequena faixa de terra irrigada, onde se encontram caramujos Biomphalaria glabrata. Dos 1.532 habitantes, 1.105 (72,1\%) submeteram-se ao exame clínico e, destes, 1.058 (95,7\%) fizeram exame parasitológico de fezes. A prevalência da esquistossomose foi de $73,1 \%$, sendo que $16,2 \%$ destes eliminavam mais de 1.000 ovos por grama de fezes. O exame clínico mostrou que o lobo esquerdo do fígado estava aumentado e/ou endurecido em $54 \%$ dos pacientes e o baço foi palpado em 21,8\%. Foram classificados como hepatosplênicas $9,8 \%$ dos examinados e como portadores da forma hepatintestinal avançada 3,7\%. Houve relação direta entre estas formas clínicas da doença e a intensidade da carga parasitária acima de 1.000 ovos de S. mansoni por grama de fezes.
\end{abstract}

Palavras-chaves: Esquistossomose. Hepatosplenomegalia. Carga parasitária.

Abstract The study was carried out in Taquarendi (Bahia), a caatinga zone with a small irrigated strip of land, where Biomphalaria glabrata snails are found. From the 1,532 inhabitants, 1,105 (72.1\%) were submitted to clinical examination and 1058 out of them (95.7\%) made stool examinations. Prevalence of schistosomiasis was $73.1 \%$ and $16.2 \%$ of these patients eliminated more than 1,000 eggs per gram of stool. By clinical examination, the size and the consistency of the left liver lobe were increased in 54\% of the individuals and the spleen was palpable in $21.8 \%$. The diagnosis of hepatosplenomegaly and of the advanced hepatointestinal clinical form was made, respectively, in $9.8 \%$ and $3.7 \%$. A direct relationship between such clinical forms of the disease and the worm load over $1.000 \mathrm{~S}$. mansoni eggs/g of fezes was found.

Key-words: Schistosomiasis. Hyperendemic area. Worm load.

A esquistossomose mansoni é considerada uma das doenças endêmicas mais importantes e mais difundidas do mundo, estimando-se que infecte cerca de 200 milhões de indivíduos. No Brasil, antes da implantação do Programa Especial de Controle da Esquistossomose (PECE), a esquistossomose atingia entre dez e doze milhões de pessoas ${ }^{33}$, sendo considerada uma das mais importantes áreas de ocorrência da doença ${ }^{35}$.

Os estudos de campo em diversas áreas endêmicas do Brasil, e posteriormente em outros países, têm contribuído para a compreensão da história natural da doença ${ }^{9}$, de sua epidemiologia ${ }^{25}$, evolução das formas clínicas ${ }^{11}{ }^{12}$, tais como o desenvolvimento da forma hepatoesplênica ${ }^{40}$, suas relações com a carga parasitária ${ }^{202331}$, reinfecções sucessivas ${ }^{17}$, características imunológicas do hospedeiro ${ }^{13}{ }^{14}$, caracteres raciais ${ }^{10}$, terapêutica específica ${ }^{56819} \mathrm{e}$ controle ${ }^{78213041}$. Muitos desses aspectos ainda não estão completamente esclarecidos.

O presente trabalho, parte integrante do Projeto sobre Esquistossomose na Chapada Diamantina (PECD) apresenta dados sobre a infecção pelo Schistosoma mansoni e sua relação com as formas graves da doença na área hiperendêmica de Taquarendi.

1. Faculdade de Medicina da Universidade Federal da Bahia, Salvador, BA; 2. Faculdade de Medicina do Triângulo Mineiro, Uberaba, MG. Endereço para correspondência: Dr. José Carlos Bina. R. Guadalajara 430/902, Ondina, 40140-461 Salvador, BA

Telefax: 5571 247-4577.

e-mail: bina@ufba.br

Recebido para publicação em 30/11/2001. 


\section{MATERIAL E MÉTODOS}

O trabalho foi realizado no distrito de Taquarendi, pertencente ao município de Mirangaba, distando cerca de $400 \mathrm{~km}$ a noroeste de Salvador (Bahia). Embora situado numa região de caatinga, apresenta a peculiaridade de possuir um riacho perene que tem como característica o seu desaparecimento após um percurso de $6 \mathrm{~km}$. A população está agrupada em três vilarejos: a) Mandacaru, b) Taquarendi propriamente dita e c) Volta da Serra, vivendo, aproximadamente em 400 casas. Valas de irrigação tornam fértil uma faixa de terra de 300 metros de largura, onde se cultivam, principalmente, alho, feijão e frutas. A maioria da população é autóctone e vive às margens da área irrigada, em estreito contato com o riacho e os canais de irrigação. A água que a população utiliza para banhos, lavagem de roupas e de utensílios domésticos é também a do riacho. Na época de nosso estudo, não havia serviços de água encanada e nem privadas. O vetor do Schistosoma mansoni é a espécie Biomphalaria glabrata, encontrada em grande número nas valas de irrigação durante todas as estações do ano. A população não apresenta malária, calazar, sinais evidentes de desnutrição, nem altos índices de parasitose intestinal.

A esquistossomose na localidade começou a ser estudada em 1968, através de um corte transversal, cujos resultados são mencionados no presente trabalho. $\mathrm{Na}$ época, foram feitos exames parasitológicos de fezes, qualitativo e quantitativo, e exame clínico da população. Os exames de fezes para o diagnóstico da esquistossomose foram realizados pelos métodos qualitativo de Lutz $^{32}$ e quantitativo de Stoll ${ }^{44}$. Os exames clínicos constaram da palpação do fígado e do baço, assinalados como impalpável, palpável à inspiração simples, profunda, ao nível ou abaixo do rebordo costal.
Neste último caso, medido em $\mathrm{cms}$. As medidas do fígado eram tomadas no rebordo costal, na linha médioclavicular, para o lobo direito, ao nível do apêndice xifóide para o lobo esquerdo do órgão. Além do tamanho, anotava-se a consistência (flácida, levemente endurecida ou dura) e, também, a superfície (lisa ou nodular), bem como o caráter de proeminência do lobo esquerdo (presente ou ausente). O baço era medido a partir do rebordo costal esquerdo no sentido de seu maior volume.

Consideramos como forma hepatintestinal avançada (HA), quando o paciente apresentava o fígado endurecido, quase sempre, aumentado de volume, principalmente seu lobo esquerdo, que, freqüentemente, era proeminente e com superfície nodular, e baço impalpável ou palpável somente à inspiração. Para ser hepatosplênico (HE), além do fígado com tais características, o baço era palpado no rebordo ou além deste e, geralmente, endurecido.

Como a forma hepatosplênica e a hepatintestinal avançada são mais freqüentes em indivíduos com idade menos avançada, época em que também é maior a quantidade de ovos nas fezes, para estudar a relação entre carga parasitária e formas graves da doença, fizemos o pareamento de ambas com a forma hepatintestinal $(\mathrm{HI})$ (diferença máxima de cinco anos na maioria das vezes).

Análise estatística. $O$ processamento e a análise estatística dos dados foram feitos utilizando-se o Statistical Package for Social Sciences (SPSS), para construção do banco de dados, tabelas, cruzamentos de variáveis e o teste de linearidade. Considerou-se como significante, o $\mathrm{p}<0,05$, ou seja, quando a probabilidade de que a associação encontrada pudesse ter ocorrido ao acaso, fosse inferior a 5\%.

\section{RESULTADOS}

Dos 1.532 habitantes de Taquarendi, 1.105 (72,1\%) submeteram-se ao exame clínico e destes, 1.058 (95,7\%) fizeram o exame parasitológico de fezes. Na população havia $567(51,3 \%)$ pessoas do sexo masculino e 538 $(48,7 \%)$ do feminino. Quanto à composição racial, 320 $(29,0 \%)$ eram brancos, 17 (1,5\%) negros e 767 (69,5\%) mulatos. Em relação à idade, havia predomínio dos mais jovens, sendo que $648(58,6 \%)$ estavam abaixo dos 20 anos de idade (Tabela 1).

A intensidade da infecção avaliada de acordo com o a idade é mostrada na Figura 1. Após um exame de fezes qualitativo e outro quantitativo, realizados na

Tabela 1 - População de Taquarendi (BA) em relação à idade.

\begin{tabular}{lrr}
\hline Idade (anos) & \multicolumn{2}{c}{ ovos } \\
\cline { 2 - 3 } & $\mathrm{n}^{\circ}$ & $(\%)$ \\
\hline $0-9$ & 367 & 33,2 \\
$10-19$ & 281 & 25,4 \\
$20-29$ & 179 & 16,2 \\
$\geq 30$ & 278 & 25,2 \\
\hline Total & 1.105 & 100,0 \\
\hline
\end{tabular}

mesma amostra de fezes de 1.058 indivíduos, havia $773(73,1 \%)$ positivos, sendo $125(16,2 \%)$ com mais de 1.000 ovos por grama de fezes. Em relação à população total examinada, $11,7 \%$ eliminavam mais de 1.000 ovos por grama de fezes. A média geométrica do número de ovos por grama de fezes nas pessoas com exame de fezes positivo foi de 309 , sendo mais elevada no sexo feminino.

Ao exame clínico, o lobo esquerdo do fígado foi palpado abaixo do apêndice xifóide em 597 (54,0\%) dos indivíduos. O baço foi palpado em 241 (21,8\%) de 1.105 indivíduos. Quanto às formas clínicas, $108(9,8 \%)$ indivíduos foram considerados como tendo a forma hepatosplênica da doença, $41(3,7 \%)$ a hepatintestinal avançada e os $909(86,5 \%)$ restantes tinham a forma hepatintestinal.

Quanto ao sexo, dos 108 hepatosplênicos 46 $(42,5 \%)$ eram homens e $62(57,5 \%)$ mulheres. Entre os $41 \mathrm{com}$ a forma hepatintestinal avançada havia 23 $(56,1 \%)$ homens e $18(43,9 \%)$ mulheres. A idade dos indivíduos com a forma hepatosplênica, variou de 5 a 


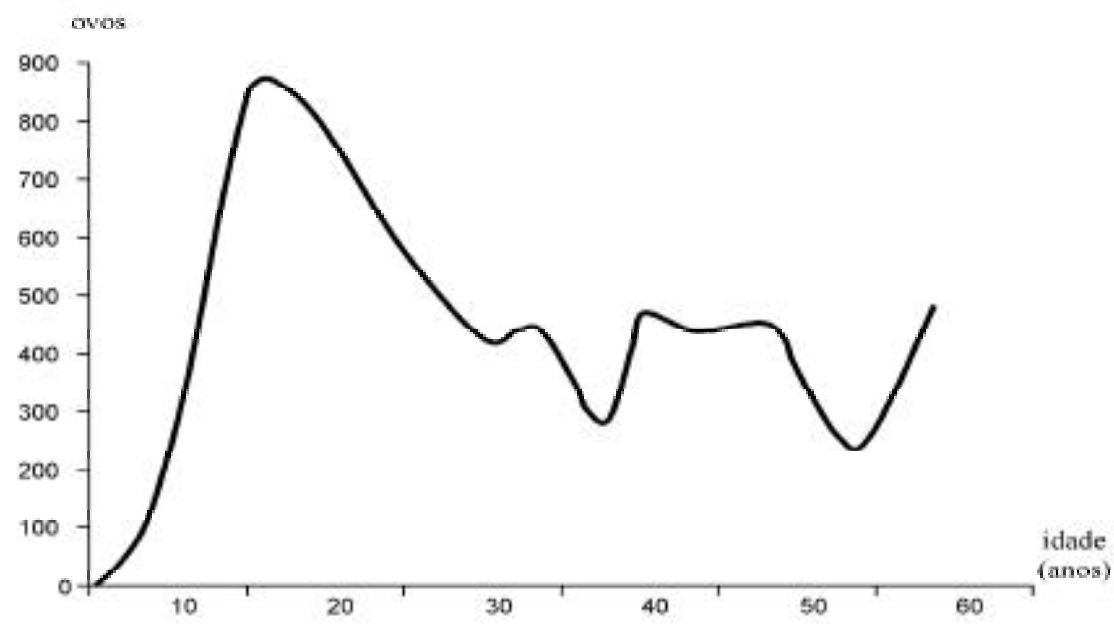

Figura 1 - Quantidade de ovos de Schistosoma mansoni eliminados por grama de fezes, de acordo com a idade, em Taquarendi, BA.

73 anos, sendo nos homens mais freqüente no grupo etário entre 10 e 30 anos (Tabela 2) e nas mulheres entre 30 e 50 anos.

Em relação à forma hepatintestinal avançada, também, as mulheres têm maior idade que os homens (Tabela 3).

\begin{tabular}{|c|c|c|c|}
\hline \multirow[b]{2}{*}{ Idade (anos) } & \multicolumn{2}{|c|}{ Sexo } & \multirow[b]{2}{*}{ Total $\left(n^{\circ}\right)$} \\
\hline & $M$ & $\mathrm{~F}$ & \\
\hline $5-9$ & 1 & 0 & 1 \\
\hline $10-14$ & 7 & 8 & 15 \\
\hline $15-19$ & 8 & 7 & 15 \\
\hline $20-24$ & 9 & 6 & 15 \\
\hline $25-29$ & 9 & 3 & 12 \\
\hline $30-39$ & 6 & 16 & 22 \\
\hline $40-49$ & 5 & 13 & 18 \\
\hline $50-59$ & 0 & 5 & 5 \\
\hline$\geq 60$ & 1 & 4 & 5 \\
\hline Total & 46 & 62 & 108 \\
\hline
\end{tabular}

Quando na população geral comparamos a intensidade da infecção, avaliada pelo número de ovos por grama de fezes, e as formas clínicas da doença (Tabela 4) observamos uma relação direta entre maior número de ovos de $S$. mansoni nas fezes, a forma hepatointestinal avançada e a forma hepatosplênica.

Tabela 3 - Idade e sexo dos indivíduos com forma hepatintestinal avançada.

\begin{tabular}{lrrr} 
& \multicolumn{2}{c}{ Sexo } & \\
\cline { 2 - 3 } Idade (anos) & $\mathrm{M}$ & $\mathrm{F}$ & Total $\left(\mathrm{n}^{\circ}\right)$ \\
\hline $5-9$ & 4 & 0 & 4 \\
$10-14$ & 11 & 4 & 15 \\
$15-19$ & 2 & 1 & 3 \\
$20-24$ & 2 & 0 & 2 \\
$25-29$ & 0 & 3 & 3 \\
$30-39$ & 1 & 0 & 1 \\
$40-49$ & 1 & 4 & 5 \\
$50-59$ & 1 & 2 & 3 \\
$\geq 60$ & 1 & 4 & 5 \\
\hline Total & 23 & 18 & 41
\end{tabular}

Tabela 4 - Relação entre o número de ovos do S. mansoni por grama de fezes e as formas clínicas, em Taquarendi.

\begin{tabular}{|c|c|c|c|c|c|c|c|}
\hline \multicolumn{8}{|l|}{$\begin{array}{l}\text { Intensidade } \\
\text { da infecção }\end{array}$} \\
\hline (no ovos & & & orma & línicas & & & \\
\hline \multirow[t]{2}{*}{ g fezes) } & \multicolumn{2}{|c|}{$\mathrm{HI}$} & \multicolumn{2}{|c|}{$\mathrm{HA}$} & \multicolumn{2}{|c|}{$\mathrm{HE}$} & Total \\
\hline & $\mathrm{n}^{0}$ & $\%$ & $\mathrm{n}^{0}$ & $\%$ & $\mathrm{n}^{\circ}$ & $\%$ & \\
\hline Negativos & 270 & 94,8 & 1 & 0,3 & 14 & 4,9 & 285 \\
\hline $1-99$ & 126 & 82,3 & 9 & 5,9 & 18 & 11,8 & 153 \\
\hline $100-499$ & 208 & 83,5 & 5 & 2,0 & 36 & 14,5 & 249 \\
\hline $500-999$ & 180 & 84,5 & 10 & 4,7 & 23 & 10,8 & 213 \\
\hline$\geq 1.000$ & 125 & 79,2 & 16 & 10,1 & 17 & 10,7 & 158 \\
\hline Total & 909 & 85,9 & 41 & 3,9 & 108 & 10,2 & 1.058 \\
\hline
\end{tabular}

$\mathrm{X}_{4}^{2}$ para a forma $\mathrm{HA}=31,3, \mathrm{p}<0,0005$

$\mathrm{X}_{4}{ }^{2}$ para a forma $\mathrm{HE}=15, \mathrm{p}<0,005$ 
A Tabela 5 compara a intensidade da infecção nas formas clínicas hepatintestinal e hepatintestinal avançada, nos pacientes pareados em relação à idade. Observamos que esta última forma clínica está associada, de forma significante com as cargas parasitárias mais elevadas, a partir de 1.000 ovos por grama de fezes $(p<0,004)$. Do mesmo modo, quando comparamos a forma hepatosplênica com a forma hepatintestinal, a associacão entre cargas parasitárias mais elevadas(acima de 1000 ovos g/fezes) e a forma hepatosplênica também ocorre com significância estatística de $p<0,05$ (Tabela 6).

A análise dos 8 pacientes com mais de 2.000 ovos por grama de fezes, nestes 298 pareados, mostrou que $7(87,5 \%)$ deles eram hepatosplênicos ou hepatintestinais avançados.

Tabela 5 - Relação entre intensidade da infecção e formas clínicas hepatointestinal e hepatointestinal avançada, em relação à idade em pacientes pareados. Taquarendi - BA.

\begin{tabular}{|c|c|c|c|c|c|c|}
\hline \multirow{4}{*}{$\begin{array}{l}\text { Intensidade da } \\
\text { infecção (no de ovos/ } \\
\text { g fezes) }\end{array}$} & \multicolumn{5}{|c|}{ Idade x Forma clínica } & \multirow{4}{*}{ Tota } \\
\hline & $0-9$ & 10-19 & $20-29$ & $30-39$ & $40 \geq$ & \\
\hline & $\mathrm{HA} / \mathrm{HI}$ & $\mathrm{HA} / \mathrm{HI}$ & $\mathrm{HA} / \mathrm{HI}$ & $\mathrm{HA} / \mathrm{HI}$ & $\mathrm{HA} / \mathrm{HI}$ & \\
\hline & $\mathrm{N}(\%)$ & $\mathrm{N}(\%)$ & $\mathrm{N}(\%)$ & $\mathrm{N}(\%)$ & $\mathrm{N}(\%)$ & \\
\hline Negativo & $1(25,0) / 1(25,0)$ & $0(0,0) / 1(6,2)$ & $0(0,0) / 0(0,0)$ & $0(0,0) / 0(0,0)$ & $0(0,0) / 4(30,8)$ & $1 / 6$ \\
\hline $1-99$ & $1(25,0) / 3(75,0)$ & $2(12,5) / 1(6,2)$ & $2(28,6) / 2(28,6)$ & $1(100,0) / 1(100,0)$ & $3(23,0) / 3(23,0)$ & $9 / 10$ \\
\hline $100-499$ & $0(0,0) / 0(0,0)$ & $1(6,2) / 5(31,3)$ & $2(28,6) / 2(28,6)$ & $0(0,0) / 0(0,0)$ & $2(15,4) / 5(38,4)$ & $5 / 12$ \\
\hline $500-999$ & $0(0,0) / 0(0,0)$ & $5(31,3) / 6(37,5)$ & $1(14,2) / 3(42,8)$ & $0(0,0) / 0(0,0)$ & $4(30,8) / 1(7,6)$ & $10 / 10$ \\
\hline$\geq 1.000$ & $2(50,0) / 0(0,0)$ & $8(50,0) / 3(18,8)$ & $2(28,6) / 0(0,0)$ & $0(0,0) / 0(0,0)$ & $4(30,8) / 0(0,0)$ & $16 / 3$ \\
\hline Total & $4 / 4$ & $16 / 16$ & $7 / 7$ & $1 / 1$ & $13 / 13$ & $41 / 41$ \\
\hline
\end{tabular}

$\mathrm{X}_{4}^{2}=15,40, \mathrm{p}<0,004$

Tabela 6 - Relação entre intensidade da infecção e formas clínicas hepatoesplênica e hepatointestinal, em relação à idade em pacientes pareados. Taquarendi-BA.

\begin{tabular}{|c|c|c|c|c|c|c|}
\hline \multirow{4}{*}{$\begin{array}{l}\text { Intensidade da } \\
\text { infecção ( } n^{\circ} \text { de ovos/ } \\
\text { g fezes) }\end{array}$} & \multicolumn{5}{|c|}{ Idade x Forma Clínica } & \multirow{4}{*}{ Total } \\
\hline & $0-9$ & $10-19$ & $20-29$ & $30-39$ & $40 \geq$ & \\
\hline & $\mathrm{HE} / \mathrm{HI}$ & $\mathrm{HE} / \mathrm{HI}$ & $\mathrm{HE} / \mathrm{HI}$ & $\mathrm{HE} / \mathrm{HI}$ & $\mathrm{HE} / \mathrm{HI}$ & \\
\hline & $\mathrm{N}(\%)$ & $\mathrm{N}(\%)$ & $\mathrm{N}(\%)$ & $\mathrm{N}(\%)$ & $\mathrm{N}(\%)$ & \\
\hline Negativo & $0(0,0) / 1(100,0)$ & $1(3,3) / 2(6,6)$ & $6(22,2) / 6(22,2)$ & $1(5,0) / 5(25,0)$ & $6(20,0) / 9(30,0)$ & $14 / 23$ \\
\hline $1-99$ & $1(100,0) / 0(0,0)$ & $5(16,7) / 6(20,0)$ & $3(11,1) / 4(14,9)$ & $3(15,0) / 3(15,0)$ & $6(20,0) / 6(20,0)$ & $18 / 19$ \\
\hline $100-499$ & $0(0,0) / 0(0,0)$ & $11(36,7) / 5(16,7)$ & $9(33,3) / 10(37,0)$ & $8(40,0) / 10(50,0)$ & $8(26,7) / 7(23,3)$ & $36 / 32$ \\
\hline $500-999$ & $0(0,0) / 0(0,0)$ & $5(16,7) / 13(43,3)$ & $5(18,5) / 6(22,2)$ & $6(30,0) / 0(0,0)$ & $7(23,3) / 8(26,7)$ & $23 / 27$ \\
\hline$\geq 1.000$ & $0(0,0) / 0(0,0)$ & $8(26,5) / 4(13,4)$ & $4(14,9) / 1(3,7)$ & $2(10,0) / 2(10,0)$ & $3(10,0) / 0(0,0)$ & $17 / 7$ \\
\hline Totais & $1 / 1$ & $30 / 30$ & $27 / 27$ & $20 / 20$ & $30 / 30$ & $108 / 108$ \\
\hline
\end{tabular}

$\mathrm{X}_{2}{ }^{2}=5,97, \mathrm{p}<0,05$

\section{DISCUSSÃO}

A prevalência de $73,1 \%$ e o percentual de $16,2 \%$ de indivíduos eliminando acima de 1.000 ovos de Schistosoma mansoni por grama de fezes demonstram que Taquarendi é uma área hiperendêmica de esquistossomose, correspondendo a uma elevada prevalência de hepatomegalia (54\%), de esplenomegalia $(21,8 \%)$ e da forma hepatosplênica $(9,8 \%)$.

Ainda hoje se discute a importância da carga parasitária no determinismo da forma hepatosplênica da esquistossomose ${ }^{39}$. Os trabalhos pioneiros de Pessoa \& Coutinho ${ }^{38}$, Pessoa \& Barros ${ }^{37}$ e Pessoa \& Amorim ${ }^{36}$ na década de cinqüenta, Kloetzel 2224252627 , Cheever ${ }^{15}$, Cheever \& Dewitt ${ }^{16}$, Barreto \& Loureiro ${ }^{3}$, Barreto \& cols $^{4}$ e Lima e Costa \& $\operatorname{cols}^{29}{ }^{30}$, já chamavam a atenção para a provável relação entre a intensidade da infecção e a forma hepatosplênica. A partir da década de oitenta, trabalhos prospectivos vêm correlacionando, além da intensidade da carga parasitária com a gravidade da doença, também persistência, ou regressão da forma hepatosplênica, na dependência de infecções mais ou menos intensas ${ }^{34} 4243$. Para alguns autores, a intensidade da infecção somada ao padrão de vida das populações, constituíriam fatores agravantes importantes no desenvolvimento das formas graves da doença 2934 . Para Barreto \& Loureiro ${ }^{3}$, a intensidade da infeccãa é um indicador de morbidade melhor do que a prevalência.

Os dados do presente trabalho mostram associação entre a elevada carga parasitária, principalmente acima de 1.000 ovos por grama de fezes, e o desenvolvimento da forma hepatosplênica e da hepatointestinal avançada. Nas áreas hiperendêmicas, entretanto, dos indivíduos que eliminam grandes quantidades de ovos de $S$. mansoninas fezes, apenas pequena proporção desenvolve a forma hepatosplênica, sugerindo que, embora a maior intensidade da infecção seja fator importante, não é o único responsável pelo determinismo das formas graves, especialmente a forma hepatosplênica da doença. 


\section{REFERÊNCIAS BIBLIOGRÁFICAS}

1. Amaral RS, Porto MAS. Evolução e situação atual do controle da esquistossomose no Brasil. Revista da Sociedade Brasileira de Medicina Tropical 27:73-90, 1994.

2. Barreto ML. Geographical and socioeconomic factors relating to the distribution of Schistosoma mansoni infection in an urban area of northeast Brazil. Bulletin of the World Health Organization 69:93-102, 1991.

3. Barreto ML, Loureiro S. The effect of Schistosoma mansoni infection on child morbidity in the state of Bahia, Brazil. Revista do Instituto de Medicina Tropical de São Paulo 26:230-235, 1984.

4. Barreto ML, Loureiro S, Melo AS, Anjos CDF. The effect of Schistosoma mansoni infection on child morbidity in the state of Bahia, Brazil. II. Analysis at the individual level. Revista do Instituto de Medicina Tropical de São Paulo 27:167-171, 1985.

5. Bina JC. Influência da terapêutica específica na evolução da esquistossomose. Dissertação de Mestrado. Universidade Federal da Bahia. Salvador, BA, 1977.

6. Bina JC. Specific therapy in the control of schistosomiasis. Memórias do Instituto Oswaldo Cruz 87:195-201, 1984.

7. Bina JC, Prata A. An attempt to control schistosomiasis mansoni in an endemic area by the use of hycanthone as chemotherapeutic agent. Revista da Sociedade Brasileira de Medicina Tropical 8:217-222, 1974.

8. Bina JC, Prata A. Regressão da hepatoesplenomegalia pelo tratamento específico da esquistossomose. Revista da Sociedade Brasileira de Medicina Tropical 16:213-218, 1983.

9. Bina JC, Prata A. A evolução natural da esquistossomose em uma área endêmica. In: Aspectos peculiares da infecção por Schistosoma mansoni. Centro Didático e Editorial da Universidade Federal da Bahia, p.13-33, 1984.

10. Bina JC, Tavares-Neto J, Prata A, Azevedo ES. Greater resistance to development of severe schistosomiasis in Brazilian Negroes. Human Biology 50:41-49, 1978.

11. Brener Z, Mourão OG. Inquérito clínico-epidemiológico em focos endêmicos de esquistossomose mansoni em Minas Gerais. Revista Brasileira de Malariologia e Doenças Tropicais 8:519-526, 1956.

12. Brener Z, Mourão OG. Observações sobre a forma hepatoesplênica da esquistossomose mansoni em Minas Gerais. Revista Brasileira de Malariologia e Doenças Tropicais 8:511517, 1956.

13. Brujning CFA. Immunology of schistosomiasis. Acta Leidensia 36:38-44, 1968

14. Butterworth BA, Dalton PR, Dune DW, Mugambi M, Ouma JH, Richardson BA, Arap Siongok TK, Sturrock RF. Immunity after treatment of human schistosomiasis mansoni. I. Study design, pretreatment observations and the results of treatment. Transaction of the Royal Society of Tropical Medicine and Hygiene 78:108-123, 1984.

15. Cheever AW. Quantitative comparison of the intensity of Schistosoma mansoni infections in man and experimental animals. Transaction of the Royal Society of Tropical Medicine and Hygiene 63:781-795, 1969.

16. Cheever AW, Dewitt WB. Repeated infection and treatment of mice with Schistosoma mansoni: functional, anatomic and immunologic observations. American Journal of Tropical Medicine and Hygiene 17:239-253, 1965.

17. Coura JR. Follow-up of patients with schistosomiasis living in non-endemic area in Brazil. Brasília Médica 11:45-47, 1975.

18. Coura Filho P, Rocha RS, Lima e Costa MFF, Katz N. A municipal level approach to the management of schistosomiasis control in Peri-Peri, MG, Brazil. Revista do Instituto de Medicina Tropical de São Paulo 34:543-548, 1992.

19. Dietze R, Prata A. A rate of reversion of hepatosplenic schistosomiasis after specific therapy. Revista da Sociedade Brasileira de Medicina Tropical 19:69-73, 1986.

20. Guyatti HL, Smith T, Gryseels B, Lengeler $C$, Mshinda $H$, Siziya $\mathrm{S}$, Salanave B, Mohome N, Makwala J, Ngimbi KP. Aggregation in schistosomiasis comparison of the relationships between prevalence and intensity in different endemic areas. Parasitology 109:45-55, 1994.

21. Katz N. Experiências com quimioterapia em grande escala no controle da esquistossomose no Brasil. Revista do Instituto de Medicina Tropical de São Paulo 22:40-51,1980.

22. Kloetzel K. Sobre a conveniência da quimioterapia da esquistossomose em população em contínuo contato com os focos. Revista do Instituto de Medicina Tropical de São Paulo 5:106-110, 1963.

23. Kloetzel K. Some quantitative aspects of diagnosis and epidemiology in schistosomiasis mansoni. American Journal of Tropical Medicine and Hygiene 12:334-337, 1963.

24. Kloetzel K. A suggestion for the prevention of severe clinical forms of schistosomiasis mansoni. Bulletin of the World Health Organization 37:686-687, 1967.

25. Kloetzel K. Alguns novos conceitos sobre a epidemiologia da esquistossomose. O Hospital 55:1471-1482, 1969.

26. Kloetzel K. Reinfection after treatment of schistosomiasis environment or predisposition. Revista do Instituto de Medicina Tropical de São Paulo 32:138-146, 1990.

27. Kloetzel K. Some personal views on the control of Schistosoma mansoni. Memórias do Instituto Oswaldo Cruz 87:221-226, 1992.

28. Lehman JS, Mott KE, Morrow RH, Muniz TM, Boyer MH. The intensity and effects of infection with Schistosoma mansoni in a rural community in North-east Brazil. American Journal of Tropical Medicine and Hygiene 25:285-294, 1976.

29. Lima e Costa MFF, Magalhães MHA, Rocha RS, Antunes CMF, Katz N. Water-contact patterns and socioeconomic variables in the epidemiology of schistosomiasis mansoni in an endemic area in Brazil. Bulletin of the World Health Organization 65:57-66, 1987.

30. Lima e Costa MFF, Rocha RS, Coura Filho P, Katz N. A 13-years follow-up of treatment and snail control in an area endemic for Schistosoma mansoni in Brazil: incidence of infection and reinfection. Bulletin of the World Health Organization 71:197-205, 1993.

31. Lima e Costa MFF, Rocha RS, Katz N. Splenomegaly in relation to Schistosoma mansoni egg counts: a population based study. Revista do Instituto de Medicina Tropical de São Paulo 28:389394, 1986.

32. Luiz A. O Schistosoma e a schistosomatose segundo observações feitas no Brasil. Memórias do Instituto Oswaldo Cruz 11:121-150, 1919. 
33. Ministério da Saúde. Programa Especial de Controle da Esquistossomose (PECE). In: VI Conferência Nacional de Saúde. Brasília, 1977.

34. Mota E, Sleigh AC. Water-contact patterns and Schistosoma mansoni infection in a rural community in northeast Brazil. Revista do Instituto de Medicina Tropical de São Paulo 29:230-235, 1984.

35. Organização Mundial da Saúde. O controle da esquistossomose: Segundo Relatório do Comitê de Especialistas da OMS. Traduzido por Maria de Fátima de Azevedo. Rio de Janeiro. FIOCRUZ, 1994.

36. Pessoa SB, Amorim JP. Contribuição à história natural da esquistossomose mansônica no nordeste brasileiro e sugestões para a sua profilaxia. Revista Brasileira de Malariologia e Doenças Tropicais 9:5-18, 1957.

37. Pessoa SB, Barros PL. Notas sobre a epidemiologia da esquistossomose mansônica no Estado de Sergipe. Revista de Medicina e Cirurgia de São Paulo 13:147-154, 1953.

38. Pessoa SB, Coutinho JOA. A esquistossomose mansônica como doença do trabalho. O Hospital 43:429-436, 1953.
39. Prata A. Influence of the host related factors in the development of the hepatosplenic form of schistosomiasis mansoni. Memórias do Instituto Oswaldo Cruz 87:39-44, 1992.

40. Prata A, Bina JC. Development of hepatosplenic form of schistosomiasis. Gazeta Médica da Bahia 68:49-60, 1968.

41. Silveira AC. Controle da esquistossomose no Brasil. Memórias do Instituto Oswaldo Cruz 84:91-104, 1989.

42. Sleigh AC, Hoff R, Mota EA, Sherlock I, Mott KE, Barreto ML, Maguire JH, Weller TH. Three-year prospective study of the evolution of Manson's schistosomiasis in northeast Brazil. Lancet 2:63-66, 1985.

43. Sleigh AC, Mott KE, Hoff R, Maguire JH, França Silva JT. Manson's schistosomiasis in Brazil: 11-year evaluation of successful disease control with oxamniquine. Lancet 124:59-65, 1986.

44. Stoll NR. Investigation on the control of hookworm disease. XV An effective method of counting eggs in feces. American Journal of Hygiene 3:59-70, 1923. 